\title{
PDBrt: a free database of complexes with measured drug-target residence time
}

\author{
Magdalena Lugowska ${ }^{1}$ and Marcin Pacholczyk ${ }^{1}$ \\ ${ }^{1}$ Silesian University of Technology
}

June 19, 2020

\begin{abstract}
Difficulties in translating the in vitro potency determined by cellular assays into in vivo efficacy in living organisms complicate the design and development of drugs. However, the residence time of a drug in its molecular target is becoming a key parameter in the design and optimization of new drugs, as recent studies show that residence time can reliably predict drug efficacy in vivo. Experimental approaches to binding kinetics and target ligand complex solutions are currently available, but known bioinformatics databases do not usually report information about the ligand residence time in its molecular target. To extend existing databases we developed the PDBrt database which reports ligand residence time. The PDBrt database is a free, non-commercial repository for 3D protein-ligand complex data, including the measured ligand residence time inside the binding pocket of the specific biological macromolecules as deposited in The Protein Data Bank. The database contains information about both the protein and the ligand separately, as well as the protein-ligand complex and time of the ligand residence inside the protein binding site. The PDBrt database is available at https://pdbrt.polsl.pl.
\end{abstract}

\section{Hosted file}

PDBrt_a_free_database_of_complexes_with_measured_drug__target_residence_time.pdf available at https://authorea.com/users/335061/articles/460965-pdbrt-a-free-database-of-complexeswith-measured-drug-target-residence-time 


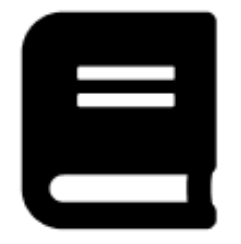

\section{Binding kinetics} data acquisition and validation

literature review to find complexes with known residence time or dissociation rate
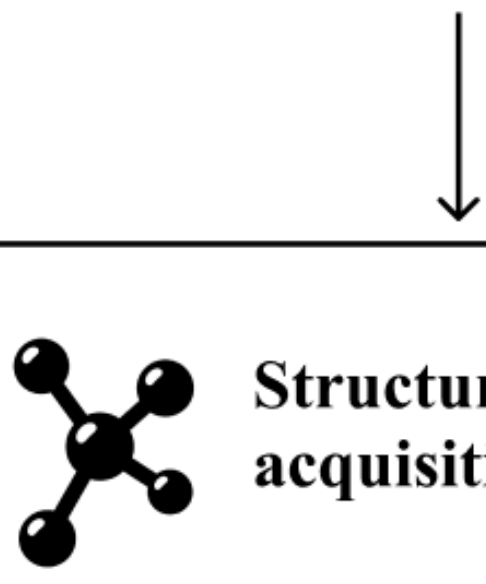

Structural data acquisition

check availability of selected complexes crystal structures

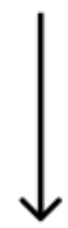

\section{PDBrt data content}

upload excel file with complex text data automatically download structure files from RCSB PDB database 


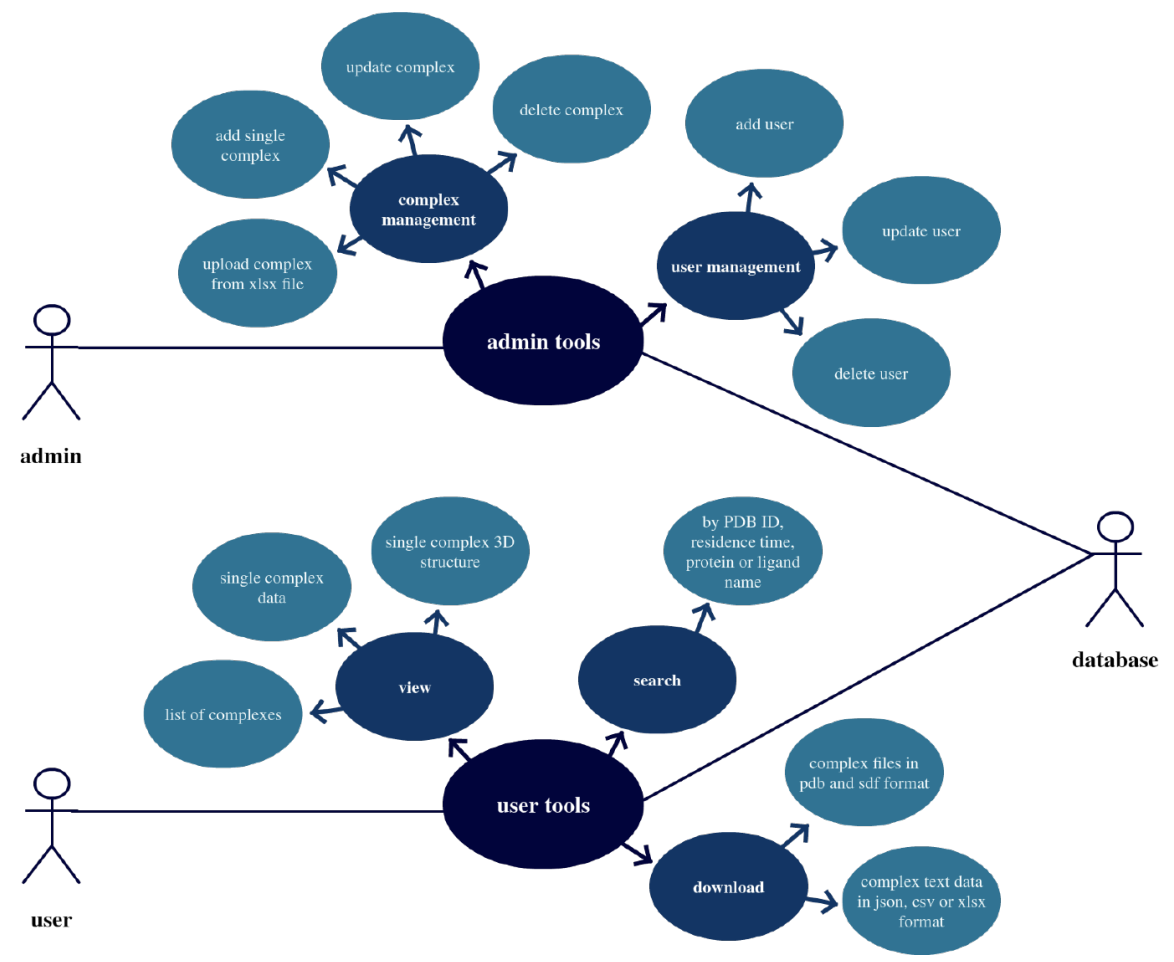




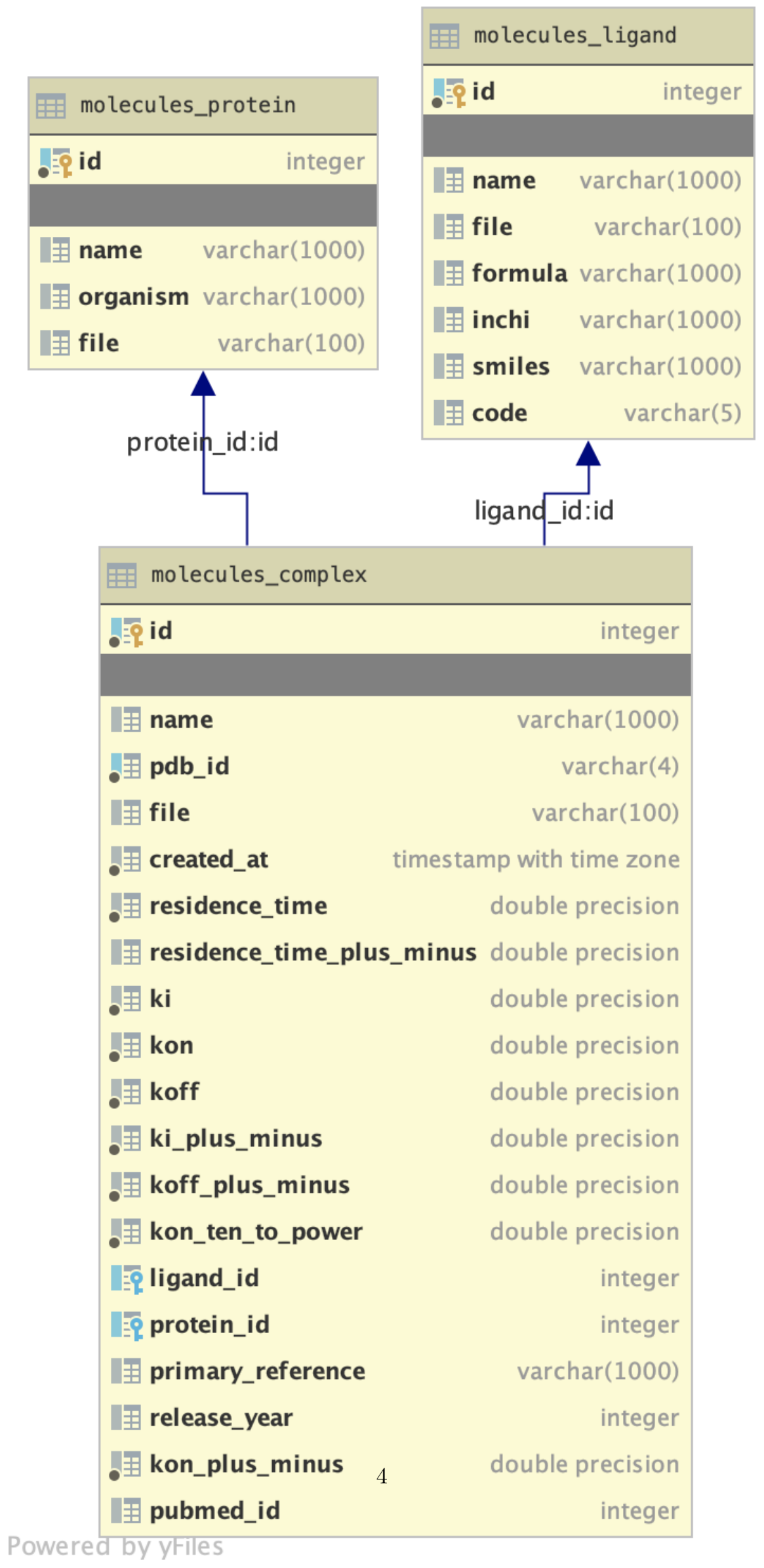

